\title{
Early onset group B streptococcal neonatal infection in Oxford 1985-96
}

\author{
Lahra M Moses, P T Heath, A R Wilkinson, H E Jeffery, D Isaacs
}

\begin{abstract}
Of 74920 babies live born in Oxford between 1985 and 1996, 41 (0.5 per 1000 95\% CI 0.4-0.7) developed definite, culture confirmed, early onset ( $<48$ hours) group $B$ streptococcal infection and $32(0.4$ per 1000 95\% CI 0.3-0.6) developed probable infection (sepsis plus colonisation). There was no significant variation in incidence with time. The mortality from definite infection was $19.5 \%$, and from probable infection $6 \%$.
\end{abstract}

These data suggest that the incidence of group B streptococcal infection in Oxford is considerably lower than that reported in the USA.

(Arch Dis Child Fetal Neonatal Ed 1998;79:F148-F149)

Keywords: group B streptococcal infection; sepsis; early onset; mortality

Group B streptococcal infection is the commonest early onset neonatal infection in industrialised countries, ${ }^{1}$ and an increasing problem in developing countries. ${ }^{2}$ In 1990 the projected incidence of early onset group B streptococcal (EOGBS) neonatal infection in the USA was 1.5 per 1000 live births, resulting in an estimated 6200 neonatal infections and 260 neonatal deaths. ${ }^{1}$ Strategies to reduce the incidence of EOGBS infection have been considered and various recommendations made. ${ }^{134}$

Very little information is available about the incidence of EOGBS infection in the United Kingdom, yet such data may be critical to decisions about which preventive strategy, if any, to adopt. In 1978-9, a laboratory based national study found 66 cases of EOGBS infection in 226899 live births, an incidence of 0.3 cases per 1000 live births. ${ }^{5}$ There have been no comparable studies since then. We present the results of a 12 year prospective study of the incidence of EOGBS neonatal infection at the regional maternity hospital in Oxford.

University of Sydney, New South Wales, Australia

L M Moses

H E Jeffery

D Isaacs

Department of

Paediatrics,

John Radcliffe

Hospital,

Oxford OX3 9DU

P T Heath

A R Wilkinson

D Isaacs

Correspondence to:

Dr Paul Heath.

Email:paul.heath@paediatrics.

ox.ac.uk

Accepted 29 March 1998

Methods born babies admitted to the neonatal unit at the John Radcliffe Hospital, Oxford, for the years 1985-96 was undertaken to determine the incidence of early onset group B streptococcal infection. Babies born at other hospitals and transferred to this hospital postnatally were not included in the study.

Maternal screening for vaginal group B streptococci is not routine, and there is no written policy for intrapartum antibiotic treatment of women with risk factors. Women who develop fever or who have prolonged rupture of membranes often receive intrapartum antibiotics.
Surface swabs (ear and throat) are taken from every baby admitted to the neonatal unit. Swabs are also collected at delivery from any baby born to a mother with a fever or if the membranes have been ruptured for over 24 hours.

Swabs are plated on to blood agar and incubated in $5 \% \mathrm{CO}_{2}$. If $\beta$ haemolytic colonies are penicillin sensitive they are serotyped to confirm group B streptococci. For sterile site isolates, an API 20 Strep kit (Biomérieux, France) is used to confirm GBS. This comprises chemical tests used to identify bacterial group and species.

Data were collected on the neonatal unit using a system that was developed and instituted in $1984 . .^{6}$ An infectious disease specialist makes a weekly ward round of all current inpatients and reviews all microbiology reports, plus the case notes of any baby with an episode of sepsis in the previous week. Episodes of confirmed and suspected infection are recorded on a standard proforma. Urinary group B streptococcal antigen testing was not used routinely during the study period.

The records of the microbiology laboratory were checked for blood and cerebrospinal fluid positive for group B streptococci to ensure that no cases of EOGBS infection had been missed.

\section{DEFINITIONS}

Definite early onset group B streptococcal infection was defined as a positive blood and/or cerebrospinal fluid culture in the context of clinical sepsis in a baby aged less than 48 hours. Probable EOGBS infection was defined as clinical signs consistent with EOGBS infection in a baby under 48 hours old, colonised with group B streptococci. ${ }^{6}$ This second category is used, as not all neonates with EOGBS infection will have positive blood or cerebrospinal fluid cultures.

Definition of definite EOGBS disease

Sepsis: a pure growth of group B streptococci from one or more blood culture bottles associated with clinical sepsis.

Meningitis: a pure growth of group B streptococci from cerebrospinal fluid or pleocytosis and positive blood cultures.
Pneumonia: respiratory distress consistent with pneumonia and the radiographic appearance of streaky opacities or confluent lobar opacification persisting for more than 24 hours, in addition to a positive blood culture for group B streptococci.

\section{Definition of probable EOGBS disease} Probable group B streptococcal pneumonia: respiratory distress consistent with pneumonia and the radiographic appearance of streaky densities or confluent lobar opacification persisting for more than 24 hours, in an infant 


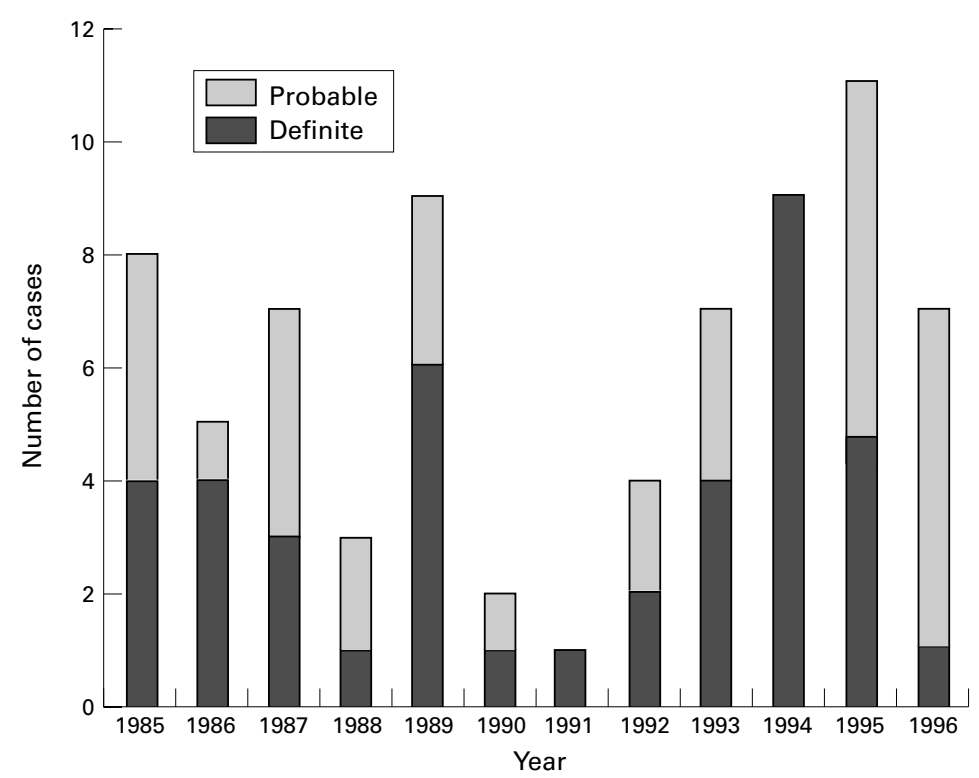

Figure 1 Number of cases of early onset group B streptococcal infection at the fohn Radcliffe Hospital, Oxford 1985-96.

colonised with group B streptococci.

Probable group $B$ streptococcal sepsis: an infant colonised with group B streptococci in the presence of two of the following clinical signs: respiratory deterioration, thrombocytopenia, bowel stasis or poor perfusion/hypotension.

\section{Results}

Over the period January 11985 to December 311996 there were 74920 live born babies at the John Radcliffe Hospital. The average annual number of live births for the 12 year period was 6243 and the average annual preterm ( $<37$ weeks of gestation) delivery rate was $7.2 \%$. Seventy three neonates had EOGBS disease, 41 positive cultures (definite infection), and 32 probable infection.

The incidence of definite EOGBS infection was 0.5 per 1000 live births (95\% CI 0.4-0.7) and the incidence of probable infection was 0.4 per 1000 live births (95\% CI 0.3-0.6). The incidence fluctuated from year to year, but there was no significant trend in rising or falling incidence over the study period (fig 1).

Ten neonatal deaths were attributed to EOGBS infection-eight (19.5\%) out of 41 with definite infection and two $(6 \%)$ out of 32 with probable infection. There was a trend to falling mortality with time: six of 19 babies $(31.6 \%)$ with definite sepsis died during 1985-90 compared with two of 22 (9\%) between 1991-6 ( $p=0.08$; Fisher's exact test).

The gestational age of definite cases varied from 24 to 42 weeks, with a mean (SD) of 35.5 (5.2) weeks. Eighteen (43.9\%) of 41 babies with definite EOGBS infection were preterm ( $<37$ weeks gestation). The mean gestational age of babies with probable EOGBS infection was 34.8 (5.4) weeks. The eight babies with definite infection and two with probable infection who died were all preterm, their gestational ages ranging from 25 to 34 weeks, with a mean of 27.5 (SD 3.4) weeks.

Of the 41 babies with definite EOGBS infection, 22 had sepsis, 13 had pneumonia, and six had meningitis. Two of the babies with meningitis died. Of the 32 babies with probable infection, 14 had pneumonia and 18 had sepsis.

\section{Discussion}

Our data suggest that the incidence of EOGBS infection in Oxford is considerably lower than that in most parts of the USA, ${ }^{1}$ and that it is not rising. Our definition of EOGBS infection as occurring in the first 48 hours of life, will exclude babies aged 2-7 days with group B streptococcal infection who would be included in a broader definition of EOGBS as $<7$ days, which is sometimes used. However, there was only one baby who fell into this category, and our incidence would have been unchanged.

Our methods for detection of EOGBS infection are standard and unlikely to have missed cases of confirmed infection. Babies with "probable" infection are recorded in Oxford because of concerns about the sensitivity of systemic cultures, ${ }^{7}$ particularly after maternal intrapartum antibiotic administration. We have been insufficiently convinced of the specificity of urinary group B streptococcal antigen detection to use it to detect or define cases. If all definite and probable cases are combined, the incidence of EOGBS infection is 0.9 per 1000 live births, only half the level reported in most parts of the USA.

The mortality from EOGBS infection in Oxford over the 12 years is high, compared with current US national data. ${ }^{1}$ However, the John Radcliffe Hospital is the regional maternity referral centre for women at high risk of adverse pregnancy outcome. The babies in Oxford who died from EOGBS infection were extremely preterm; no full term baby died from EOGBS infection. There was also a trend towards decreasing mortality with time.

The population reported in this study may not be typical of the British population, and it may well be that the incidence of group B streptococcal infection is different in other parts of the United Kingdom. Studies of the incidence in different regions are needed to establish a basis for trials of different strategies to reduce EOGBS infection.

We are grateful to Drs Simon Dobson, Robert Booy, Chris Richards, Mark Herbert and Fiona Shackley for their contributions to neonatal surveillance, and to the staff of the Neonatal Unit and the Oxford Public Health Laboratory Service.

1 Centers for Disease Control. Prevention of perinatal group B streptococcal disease: a public health perspective. Mortal Morbid Weekly Rep 1996; 45(RR-7): 1-24.

2 Walsh JA, Hutchins S. Group B streptococcal disease: its importance in the developing world and prospect for prevention with vaccines. Pediatr Infect Dis f 1989;8:271-6.

3 American Academy of Pediatrics. Revised guidelines for prevention of early-onset group B streptococcal (GBS) prevention of early-onset group B
infection. Pediatrics 1997;99:489-96.

4 Gilbert GL, Isaacs D, Burgess MA, et al. Prevention of neonatal group B streptococcal sepsis: is routine antenatal screening appropriate? Aust NZ $f$ Obstet Gynecol 1995;35:120-6.

5 Mayon-White RT. The incidence of GBS disease in neonates in different countries. Antibiot Chemother 1985;35:17-27.

6 Isaacs D, Moxon ER. Neonatal infections. Oxford: Butterworth-Heinemann, 1991.

7 Webber S, Lindsell D, Wilkinson AR, et al. Neonatal pneumonia. Arch Dis Child 1990;65: 207-11. 